\title{
Resultado clínico do reparo parcial nas roturas irreparáveis do manguito rotador*
}

\section{Clinical Outcome of Partial Repair of Irreparable Rotator Cuff Tears}

\author{
Eduardo Angeli Malavolta ${ }^{1}$ Luca Martinez $^{10}$ Mauro Emilio Conforto Gracitelli1 ${ }^{10}$

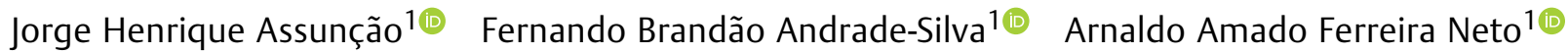 \\ 1 Grupo de Ombro e Cotovelo, Instituto de Ortopedia e \\ Traumatologia, Hospital das Clinicas HCFMUSP, Faculdade de \\ Medicina, Universidade de São Paulo, São Paulo, SP, Brasil \\ Endereço para correspondência Eduardo Angeli Malavolta, MD, PhD, \\ Rua Dr. Ovídio Pires de Campos, 333, Cerqueira Cesar, São Paulo, \\ SP, 05403-010, Brasil (e-mail: eduardomalavolta@gmail.com).
}

Rev Bras Ortop 2022;57(1):136-143.

\section{Resumo \\ Palavras-chave \\ - manguito rotador \\ - artroscopia \\ - ombro \\ - avaliação de resultado de intervenções terapêuticas}

\footnotetext{
Abstract

Trabalho desenvolvido no Instituto de Ortopedia e Traumatologia, Hospital das Clinicas HCFMUSP, Faculdade de Medicina, Universidade de São Paulo, São Paulo, SP, Brasil.
} de flexão anterior ativa. $(66,7 \%)(p=0,015)$.
Objetivo Avaliar o resultado funcional de pacientes submetidos ao reparo parcial por via artroscópica de roturas extensas do manguito rotador.

Métodos Série de casos retrospectiva, avaliando pacientes com roturas extensas do manguito rotador submetidos ao reparo parcial por via artroscópica. O desfecho primário foi a escala American Shoulder and Elbow Surgeons Standardized Shoulder Assessment Form (ASES, na sigla em inglês) aos 24 meses. Foram desfechos secundários a escala Modified-University of California at Los Angeles Shoulder Rating Scale (UCLA, na sigla em inglês), e seus subdomínios satisfação, flexão anterior ativa e força

Resultados Avaliamos 33 pacientes. A escala da ASES evoluiu de 39,7 $\pm 19,6$ para $77,6 \pm 17,4(p<0,001)$. A escala da UCLA evoluiu de $13,3 \pm 5,5$ para $27,9 \pm 5,6$ $(p<0,001)$. A taxa de satisfação foi de $97 \%$. O número de pacientes com flexão anterior ativa $>150^{\circ}$ passou de $12(36,4 \%)$ para $25(75,8 \%)(p=0,002)$. O número de pacientes com força de flexão anterior ativa normal ou boa passou de $9(27,3 \%)$ para 22

Conclusão O reparo parcial nas roturas irreparáveis do manguito rotador leva a melhora significativa de acordo com as escalas da ASES e UCLA.

Objective To evaluate the functional outcome of patients who underwent partial arthroscopic repair of massive rotator cuff tears. recebido

05 de Março de 2020

aceito

06 de Julho de 2020

Publicado on-line

Outubro 29, 2020
DOI https://doi.org/ 10.1055/s-0040-1716763. ISSN 0102-3616.
(C) 2020. Sociedade Brasileira de Ortopedia e Traumatologia. All rights reserved.

This is an open access article published by Thieme under the terms of the Creative Commons Attribution-NonDerivative-NonCommercial-License, permitting copying and reproduction so long as the original work is given appropriate credit. Contents may not be used for commercial purposes, or adapted, remixed, transformed or built upon. (https://creativecommons.org/ licenses/by-nc-nd/4.0/)

Thieme Revinter Publicações Ltda., Rua do Matoso 170, Rio de Janeiro, RJ, CEP 20270-135, Brazil 


\section{Keywords \\ - rotator cuff \\ - arthroscopy \\ - shoulder \\ - evaluation of results of therapeutic interventions}

Methods Retrospective case series evaluating patients with massive rotator cuff tears who underwent partial arthroscopic repair. The primary outcome was the American Shoulder and Elbow Surgeons Standardized Shoulder Assessment Form (ASES) at 24 months. The secondary outcomes were the Modified-University of California at Los Angeles Shoulder Rating Scale (UCLA), and the following subdomains: satisfaction, active forward flexion and strength of forward flexion subdomains.

Results We evaluated 33 patients. The ASES scale evolved from $39.7 \pm 19.6$ to $77.6 \pm 17.4(p<0.001)$. The UCLA scale evolved from $13.3 \pm 5.5$ to $27.9 \pm 5.6$ $(p<0.001)$. The satisfaction rate was $97 \%$. The number of patients with active forward flexion $>150^{\circ}$ increased from $12(36.4 \%)$ to $25(75.8 \%)(p=0.002)$. The number of patients with normal or good strength of forward flexion increased from $9(27.3 \%)$ to $22(66.7 \%)(p=0.015)$.

Conclusion Partial repair of irreparable rotator cuff tears leads to significant improvement according to the ASES and UCLA scales.

\section{Introdução}

A rotura do manguito rotador acomete $20 \%$ da população e até $50 \%$ dos pacientes $>80$ anos, ${ }^{1}$ e é responsável por $23 \%$ dos atendimentos realizados por um especialista em cirurgia do ombro. ${ }^{2}$ Entre 2003 e 2015, houve no Brasil um aumento de $238 \%$ no número de cirurgias para o reparo do manguito rotador. $^{3}$

O tratamento das roturas irreparáveis do manguito rotador é desafiador e controverso, e a literatura médica ainda não aponta para um consenso. Diversas técnicas cirúrgicas são descritas, como desbridamento, balão subacromial, tenotomia ou tenodese do bíceps, reparo parcial, interposição com enxerto, reconstrução da cápsula superior, transferência tendinosa e artroplastia reversa. ${ }^{4}$

Burkhart, ${ }^{5}$ em 1994, descreveu o conceito de rotura funcional do manguito rotador. Esta é uma rotura que apresenta deficiência anatômica com biomecânica intacta. No mesmo ano, Burkhart et al., ${ }^{6}$ relataram os resultados de uma série de 14 pacientes submetidos ao reparo parcial do manguito rotador. Nesta técnica, os autores reparam as margens da lesão, restaurando o balanço de forças e o sistema de "ponte pênsil" do ombro, sem, no entanto fechar totalmente o defeito. A elevação ativa dos pacientes evoluiu de $91^{\circ}$ para $150^{\circ}$, enquanto a escala da Modified-University of California at Los Angeles Shoulder Rating Scale (UCLA, na sigla em inglês) evoluiu de 10 para 28.

Malahias et al., ${ }^{7}$ numa revisão sistemática, demonstraram que o reparo parcial melhora significativamente a força e a pontuação das escalas funcionais. Maillot et al, ${ }^{8}$ numa metanálise, não observaram diferença entre o reparo parcial, reparo completo e artroplastia reversa.

Embora a técnica de reparo parcial por via artroscópica tenha sido descrita há 25 anos, o número de artigos sobre o tema é limitado. Uma revisão sistemática publicada em 2019 encontrou apenas 11 estudos com mínimo de 12 meses de seguimento e avaliação funcional com escalas padronizadas. ${ }^{7}$

O objetivo do presente estudo foi avaliar o resultado funcional de pacientes submetidos ao reparo parcial por via artroscópica de roturas extensas do manguito rotador.

\section{Métodos}

Realizamos uma série de casos retrospectiva, com dados colhidos prospectivamente. Os pacientes foram operados por quatro médicos pertencentes a uma mesma instituição, todos membros efetivos da Sociedade Brasileira de Cirurgia do Ombro e Cotovelo, com experiência superior a 10 anos. Os procedimentos foram realizados entre os anos de $2013 \mathrm{e}$ 2017.

Foram incluídos pacientes submetidos ao reparo parcial do manguito rotador por via artroscópica. Não foram incluídos pacientes com artrose glenoumeral na avaliação radiográfica, ou acometidos de instabilidade ou capsulite adesiva. Foram excluídos os pacientes sem avaliação clínica pré- ou pós-operatória.

O trabalho foi aprovado pelo Comitê de Ética da instituição sob o número 1142.

\section{Desfechos}

Os desfechos avaliados foram a Escala American Shoulder and Elbow Surgeons Standardized Shoulder Assessment Form (ASES, na sigla em inglês) ${ }^{9}$ (desfecho primário) e a Escala UCLA ${ }^{10}$ (desfecho secundário), realizadas uma semana antes da cirurgia e após 6, 12 e 24 meses.

As escalas foram aplicadas por uma auxiliar de pesquisa não participante do estudo.

\section{Variáveis analisadas}

Foram avaliadas as seguintes variáveis:

Referentes aos pacientes: idade, sexo, acometimento do lado dominante, tabagismo, diabetes, hipertensão arterial sistêmica, hipotireoidismo, artrite reumatoide, uso crônico de corticosteroides, cirurgia e infiltração prévias e problemas trabalhistas.

Referentes à lesão: a classificação de Fuchs et al. ${ }^{11}$ para o supraespinal, infraespinal e subescapular foi determinada pela ressonância magnética (RM) pré-operatória. As demais variáveis (retração e extensão da rotura dos tendões do manguito rotador, rotura e instabilidade da cabeça longa 


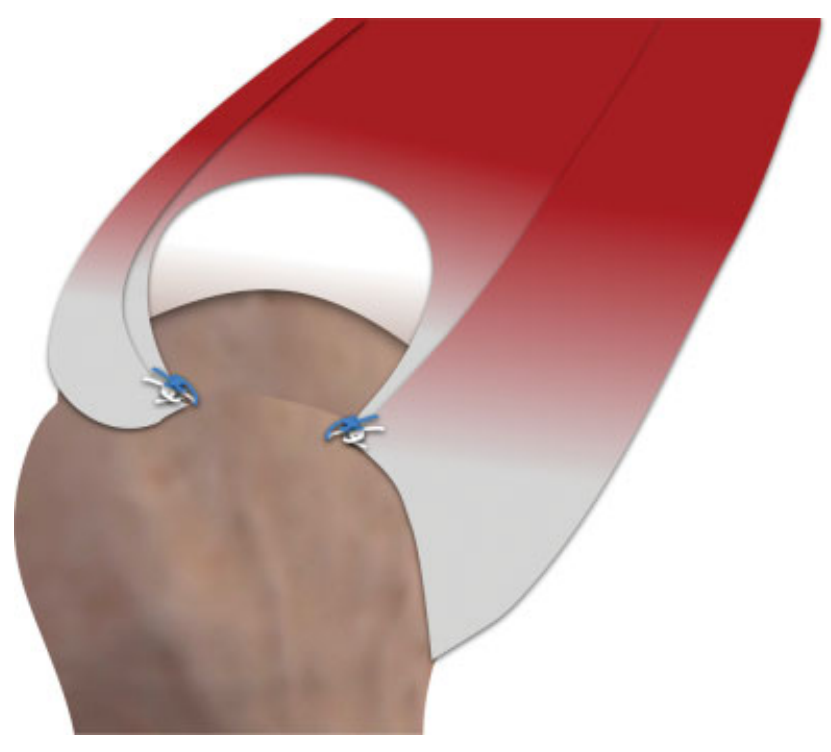

Fig. 1 Reparo parcial do manguito rotador.

do bíceps e presença de artrose glenoumeral) foram determinadas pela inspeção intraoperatória. A retração no plano coronal foi aferida de acordo com a classificação proposta por Boileau et al. ${ }^{12}$ : estádio I (retração mínima, borda da lesão lateral à superfície articular, usualmente com $<10 \mathrm{~mm}$ de retração); estádio II (retração moderada, expondo a cabeça umeral mas não a cavidade glenoidal, usualmente entre $10 \mathrm{e}$ $30 \mathrm{~mm}$ de retração); estádio III (retração grave, expondo a cavidade glenoidal, usualmente entre 30 e $50 \mathrm{~mm}$ ) e estádio IV (rotura extensa, retraídas medialmente à cavidade glenoidal).

Referentes ao procedimento: número de âncoras utilizadas, realização de acromioplastia e procedimento realizado na cabeça longa do bíceps.

\section{Intervenção}

Os procedimentos foram realizados sob anestesia geral associada ao bloqueio interescalênico. Posicionamos os pacientes em cadeira de praia ou decúbito lateral, de acordo com a preferência do cirurgião. Utilizamos os portais convencionais (posterior, anterior e lateral) e quantos mais fossem necessários.

Após inspeção detalhada e bursectomia ampla, realizamos a tração das bordas da lesão em direção ao leito ósseo, utilizando um grasper. Ao se verificar a impossibilidade de reparo completo, técnicas de mobilização tendínea foram empregadas, como capsulotomia e liberação do intervalo dos rotadores. Quando necessário, convergência de margens foi realizada. Em seguida, realizou-se o reparo tendíneo, com técnica de fileira simples, tanto do subescapular, quando lesionado, como da porção posterosuperior do manguito
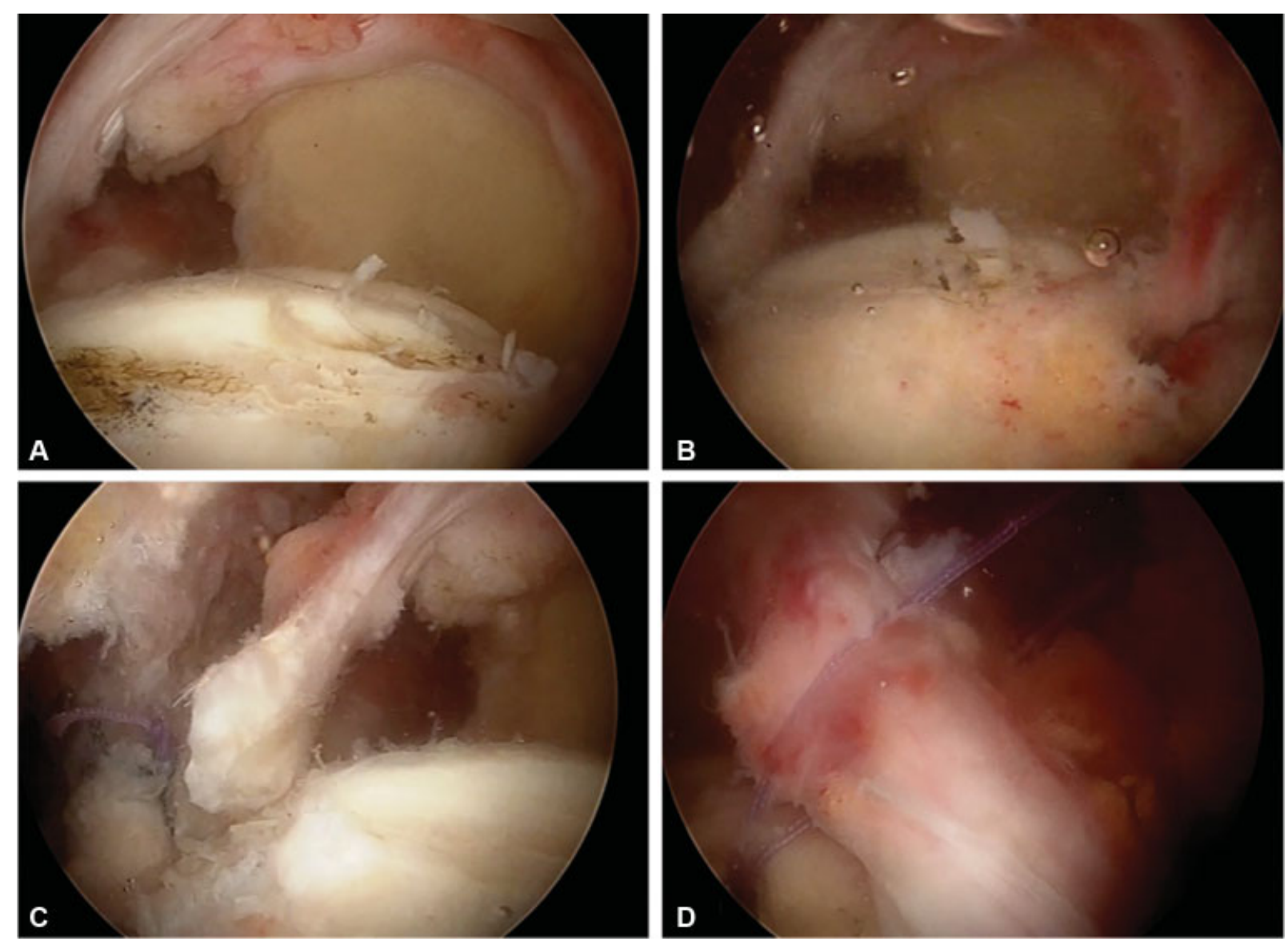

Fig. 2 Reparo parcial do manguito rotador, visão artroscópica. (A) Antes do reparo; (B) Após o reparo; (C) Sutura da região anterior (subescapular); (D) sutura da região posterior (infraespinal). 
rotador (infraespinal e supraespinal). Consideramos o procedimento como reparo parcial quando a lesão não fosse totalmente reparável, persistindo uma parte do footprint exposto. A - Figura 1 apresenta uma visão esquemática de um reparo parcial, enquanto a - Figura 2 detalha uma das cirurgias da casuística.

Caso a cabeça longa do bíceps apresentasse rotura parcial ou instabilidade, era realizada a tenotomia nos pacientes $>65$ anos ou tenodese nos demais. Acromioplastia era realizada conforme decisão do cirurgião.

\section{Reabilitação}

Os pacientes foram orientados a permanecer com tipoia com almofada abdominal por 6 semanas, retirando a mesma apenas para o banho e para a realização de movimentos com cotovelo, 3 vezes ao dia. Após 6 semanas, foi iniciada movimentação passiva e ativa. Doze semanas após a cirurgia, foram iniciados exercícios para fortalecer o manguito rotador e a musculatura estabilizadora da escápula. Todos os pacientes foram acompanhados por um fisioterapeuta da instituição, realizando sessões presenciais duas vezes por semana, e orientados aos exercícios domiciliares diários.

\section{Análise estatística}

Submetemos as variáveis contínuas à avaliação da normalidade, por meio do teste de Kolmogorov-Smirnov, e da homogeneidade, por meio do teste de Levene. Apresentamos as variáveis contínuas em média, desvio padrão (DP), mediana e intervalo interquartil (IIQ), e as categóricas em valor absoluto e percentual. A comparação entre os resultados funcionais, de acordo com as escalas da ASES e UCLA, ao longo dos diversos tempos de avaliação, foi realizada pelo teste de Friedman com ajuste de Bonferroni post-hoc. A comparação entre os subdomínios da escala da UCLA (flexão anterior ativa e força de flexão anterior ativa) entre o préoperatório e os 24 meses foi realizada pelo teste de Fisher.

Utilizamos para análise dos dados o programa IBM SPSS Statistics for Windows, versão 21.0 (IBM Corp., Armonk, NY, EUA) e nível de significância de 5\%.

\section{Resultados}

Durante o período avaliado, 439 pacientes foram submetidos a cirurgias devido a afecções do manguito rotador. Não foram incluídos aqueles submetidos ao reparo completo do manguito rotador (385) ou ao desbridamento. (12) Identificamos 42 pacientes submetidos ao reparo parcial, dos quais 9 foram excluídos por não possuírem avaliação clínica pré- ou pós-operatória, totalizando 33 pacientes para a análise dos dados.

Os pacientes apresentavam uma média de 61,4 \pm 7,3 anos na ocasião da cirurgia. As demais características gerais da amostra estão expostas na - Tabela 1.

Todos os pacientes apresentavam rotura transfixante em toda a extensão do supraespinal, e na maioria (54,5\%) retraídas até a cavidade glenoidal. Rotura do infraespinal acometia $94 \%$ da amostra, e $51 \%$ apresentavam rotura transfixante do subescapular. A degeneração gordurosa foi classi-
Tabela 1 Características gerais da amostra (variáveis referentes aos pacientes)

\begin{tabular}{|c|c|c|}
\hline & $n$ & $\%$ \\
\hline \multicolumn{3}{|l|}{ Sexo } \\
\hline Masculino & 16 & 48,5 \\
\hline Feminino & 17 & 51,5 \\
\hline \multicolumn{3}{|c|}{ Lado dominante } \\
\hline Sim & 25 & 75,8 \\
\hline Não & 8 & 24,2 \\
\hline \multicolumn{3}{|l|}{ Tabagismo } \\
\hline Tabagista & 2 & 6,1 \\
\hline Ex-tabagista & 6 & 18,2 \\
\hline Não & 25 & 75,8 \\
\hline \multicolumn{3}{|l|}{ Diabetes } \\
\hline Sim & 7 & 21,2 \\
\hline Não & 27 & 81,8 \\
\hline \multicolumn{3}{|c|}{ Hipertensão arterial sistêmica } \\
\hline Sim & 14 & 42,4 \\
\hline Não & 19 & 57,6 \\
\hline \multicolumn{3}{|c|}{ Hipotireoidismo } \\
\hline Sim & 4 & 12,1 \\
\hline Não & 29 & 87,9 \\
\hline \multicolumn{3}{|c|}{ Artrite reumatoide } \\
\hline Sim & 5 & 15,2 \\
\hline Não & 28 & 84,8 \\
\hline \multicolumn{3}{|c|}{ Uso crônico de corticoides } \\
\hline Sim & 1 & 3,0 \\
\hline Não & 32 & 97,0 \\
\hline \multicolumn{3}{|c|}{ Cirurgia prévia } \\
\hline Sim & 2 & 6,1 \\
\hline Não & 31 & 93,9 \\
\hline \multicolumn{3}{|c|}{ Infiltração prévia } \\
\hline Sim & 3 & 9,1 \\
\hline Não & 30 & 90,9 \\
\hline \multicolumn{3}{|c|}{ Problemas trabalhistas } \\
\hline Sim & 5 & 15,2 \\
\hline Não & 28 & 84,8 \\
\hline
\end{tabular}

ficada como grau III de Fuchs em 33,3\% dos pacientes para o supraespinal, $27,3 \%$ para o infraespinal e $6,1 \%$ para o subescapular (-Tabela 2). A - Tabela 3 demonstra o número de âncoras utilizadas para o reparo e os procedimentos adicionais realizados.

A escala da ASES evoluiu de $39,7 \pm 19,6$ no pré-operatório para 77,6 $\pm 17,4$ aos 24 meses $(p<0,001)$. A escala da UCLA também apresentou evolução favorável, passando de $13,3 \pm 5,5$ para $27,9 \pm 5,6(p<0,001)$. Em ambas as escalas, 
Tabela 2 Características gerais da amostra (variáveis referentes à lesão)

\begin{tabular}{|c|c|c|}
\hline & $n$ & $\%$ \\
\hline \multicolumn{3}{|l|}{ Rotura do supraespinal - Retração } \\
\hline Estádio III & 15 & 45,5 \\
\hline Estádio IV & 18 & 54,5 \\
\hline \multicolumn{3}{|c|}{ Classificação de Fuchs (supraespinal) } \\
\hline Grau I & 11 & 33,3 \\
\hline Grau II & 11 & 33,3 \\
\hline Grau III & 11 & 33,3 \\
\hline \multicolumn{3}{|l|}{ Rotura do infraespinal - Extensão } \\
\hline Íntegro & 2 & 6,1 \\
\hline Metade superior & 25 & 75,8 \\
\hline Todo o tendão & 6 & 18,2 \\
\hline \multicolumn{3}{|l|}{ Rotura do infraespinal - Retração } \\
\hline Estádio I & 10 & 30,3 \\
\hline Estádio II & 6 & 18,2 \\
\hline Estádio III & 14 & 42,4 \\
\hline Estádio IV & 3 & 9,1 \\
\hline \multicolumn{3}{|l|}{ Classificação de Fuchs (infraespinal) } \\
\hline Grau I & 14 & 42,4 \\
\hline Grau II & 10 & 30,3 \\
\hline Grau III & 9 & 27,3 \\
\hline \multicolumn{3}{|l|}{ Rotura do subescapular } \\
\hline Íntegro & 9 & 27,3 \\
\hline Parcial do $1 / 3$ superior & 7 & 21,2 \\
\hline Transfixante do $1 / 3$ superior & 6 & 18,2 \\
\hline Transfixante dos $2 / 3$ superiores & 8 & 24,2 \\
\hline Todo o tendão & 3 & 9,1 \\
\hline \multicolumn{3}{|l|}{$\begin{array}{l}\text { Classificação de Fuchs } \\
\text { (subescapular) }\end{array}$} \\
\hline Grau I & 23 & 69,7 \\
\hline Grau II & 8 & 24,2 \\
\hline Grau III & 2 & 6,1 \\
\hline \multicolumn{3}{|l|}{ Rotura da cabeça longa do bíceps } \\
\hline Íntegro & 15 & 45,5 \\
\hline Rotura parcial & 14 & 42,4 \\
\hline Rotura completa & 4 & 12,1 \\
\hline \multicolumn{3}{|l|}{$\begin{array}{l}\text { Instabilidade da cabeça } \\
\text { longa do bíceps }\end{array}$} \\
\hline Estável & 11 & 33,3 \\
\hline Instável & 9 & 27,3 \\
\hline Luxado & 10 & 30,3 \\
\hline NA & 3 & 9,1 \\
\hline \multicolumn{3}{|l|}{ Artrose glenoumeral leve } \\
\hline Sim & 5 & 15,2 \\
\hline Não & 28 & 84,8 \\
\hline
\end{tabular}

Tabela 3 Características gerais da amostra (variáveis referentes aos procedimentos)

\begin{tabular}{|l|l|l|}
\hline & $n$ & $\%$ \\
\hline Âncoras no supraespinal ou infraespinal & & \\
\hline 1 & 10 & 30,3 \\
\hline 2 & 17 & 51,5 \\
\hline$\geq 3$ & 6 & 18,2 \\
\hline Âncoras no subescapular & & \\
\hline 0 & 11 & 33,3 \\
\hline 1 & 20 & 60,6 \\
\hline 2 & 2 & 6,1 \\
\hline Acromioplastia & & \\
\hline Sim & 9 & 27,3 \\
\hline Não & 24 & 72,7 \\
\hline Procedimento na cabeça longa do bíceps & & \\
\hline Nenhum & 9 & 27,3 \\
\hline Tenotomia & 12 & 36,4 \\
\hline Tenodese & 12 & 36,4 \\
\hline
\end{tabular}

Tabela 4 Avaliação funcional pré- e pós-operatória

\begin{tabular}{|l|l|l|l|l|l|}
\hline & Média & DP & Mediana & IIQ & valor $-p$ \\
\hline UCLA & & & & & \\
\hline Pré-operatório & 13,3 & 5,5 & 12,7 & 7,5 & $p<0,001^{*}$ \\
\hline 6 meses & 27,2 & 5,8 & 28,0 & 6,5 & \\
\hline 12 meses & 28,1 & 6,4 & 29,0 & 7,0 & \\
\hline 24 meses & 27,9 & 5,6 & 29,0 & 10,5 & \\
\hline ASES & & & & & \\
\hline Pré-operatório & 39,7 & 19,6 & 37,0 & 22,9 & $p<0,001^{*}$ \\
\hline 6 meses & 69,6 & 21,2 & 73,3 & 31,5 & \\
\hline 12 meses & 75,7 & 20,5 & 80,0 & 30,0 & \\
\hline 24 meses & 77,6 & 17,4 & 83,8 & 27,6 & \\
\hline
\end{tabular}

Abreviações: ASE, Escala American Shoulder and Elbow Surgeons Standardized Shoulder Assessment Form; DP, Desvio Padrão IIQ, Intervalo Interquartil; UCLA: Escala Modified-University of California at Los Angeles Shoulder Rating Scale.

*valores referentes ao teste de Friedman.

Análise post-hoc e Bonferroni:

De acordo com a escala da UCLA, os valores pré-operatórios diferiram dos obtidos aos 6,12 e 24 meses $(p<0,001)$. As demais comparações $(6 \times 12$ meses, $6 \times 24$ meses e $12 \times 24$ meses) não apresentaram diferença estatisticamente significante ( $p=0,408,0,588$ e 0,945 , respectivamente). De acordo com a escala da ASES, os valores pré-operatórios diferiram dos obtidos aos 6,12 e 24 meses $(p<0,001)$ e entre 6 e 24 meses $(p=0,028)$. As demais comparações $(6 \times 12$ meses e $12 \times 24$ meses $)$ não apresentaram diferença estatisticamente significante $(p=0,150$ e 0,426 , respectivamente).

aos 6 meses já era observada melhora estatisticamente significativa. Os dados estão expostos na - Tabela 4.

A análise dos subdomínios da escala da UCLA demonstrou que 31 pacientes (94\%) estavam satisfeitos aos 6 e 12 meses e 
Tabela 5 Flexão anterior ativa pré- e pós-operatória

\begin{tabular}{|c|c|c|c|c|c|}
\hline & \multicolumn{4}{|c|}{ Flexão anterior ativa } & \multirow{3}{*}{ valor-p } \\
\hline & \multicolumn{2}{|c|}{$\begin{array}{l}\text { Pré-opera- } \\
\text { tório }\end{array}$} & \multicolumn{2}{|c|}{24 meses } & \\
\hline & $n$ & $\%$ & $n$ & $\%$ & \\
\hline$\geq 150^{\circ}$ & 12 & 36,4 & 25 & 75,8 & 0,002 \\
\hline $120^{\circ}-150^{\circ}$ & 11 & 33,3 & 1 & 3,0 & \\
\hline $90^{\circ}-120^{\circ}$ & 7 & 21,2 & 5 & 15,2 & \\
\hline $45-90^{\circ}$ & 1 & 3,0 & 1 & 3,0 & \\
\hline$<45^{\circ}$ & 2 & 6,1 & 1 & 3,0 & \\
\hline
\end{tabular}

Tabela 6 Força de flexão anterior ativa pré- e pós-operatória

\begin{tabular}{|l|l|l|l|l|l|}
\hline & \multicolumn{3}{|l|}{$\begin{array}{l}\text { Força de flexão anterior } \\
\text { ativa }\end{array}$} & \\
\hline & \multicolumn{2}{|l|}{$\begin{array}{l}\text { Pré- } \\
\text { operatório }\end{array}$} & \multicolumn{2}{l|}{$\mathbf{2 4}$ meses } & valor-p \\
\hline & $\mathbf{n}$ & $\%$ & $\mathbf{n}$ & $\%$ & \\
\hline Grau 5 (Normal) & 3 & 9,1 & 12 & 36,4 & 0,015 \\
\hline Grau 4 (Boa) & 6 & 18,2 & 10 & 30,3 & \\
\hline Grau 3 (Regular) & 12 & 36,4 & 7 & 21,2 & \\
\hline Grau 2 (Fraco) & 6 & 18,2 & 3 & 9,1 & \\
\hline $\begin{array}{l}\text { Grau 1 } \\
\text { (Contração } \\
\text { muscular) }\end{array}$ & 6 & 18,2 & 1 & 3,0 & \\
\hline
\end{tabular}

32 pacientes (97\%) aos 24 meses de seguimento. A flexão anterior ativa evoluiu de uma média de 2,8 para 3,9 pontos, e o número de pacientes com flexão $>150^{\circ}$ passou de 12 $(36,4 \%)$ para $25(75,8 \%)$, diferença estatisticamente significante $(p=0,002)$. Os dados estão expostos na - Tabela 5 . Dentre os pacientes, 18 melhoraram o arco de movimento, 6 pioraram e 9 não tiveram alteração. A força de flexão anterior ativa evoluiu de 3,9 para 4,5 pontos em média, e o número de pacientes com força normal ou boa passou de 9 (27,3\%) para 22 (66,7\%), também com diferença estatisticamente significante $(p=0,015)$. Os dados podem ser observados na -Tabela 6.

\section{Discussão}

Nosso estudo demonstrou uma melhora significativa de acordo com as escalas clínicas avaliadas. A escala da ASES evoluiu de 39,7 para 77,6 $(p<0,001)$ aos 24 meses, enquanto a da UCLA evoluiu de 13,3 para $27,9(p<0,001)$, demonstrando significância estatística e relevância clínica. ${ }^{13}$ Os resultados estão de acordo com outros artigos que avaliam o reparo parcial do manguito rotador. De acordo com a escala da ASES, Cuff et al. ${ }^{14}$ demonstraram progresso de 46,6 para 79,3 , enquanto Holtby et al. ${ }^{15}$ de 42,7 para 71,4 . De acordo com a escala da UCLA, Burkhart et $\mathrm{al}^{6}$ demonstraram evolução de 9,8 para 27,6 , Franceschi et al. ${ }^{16}$ de 8,6 para 28,8 e Iagulli et al. ${ }^{17}$ de 12,1 para 29,5 . Autores que utilizam a escala de Constant reportam valores pré-operatórios variando de 36,3 a 45,9, e pós-operatórios de 69,9 a 75,3. ${ }^{15,18-21}$
Até o momento, não existem estudos comparativos randomizados entre os principais métodos de tratamento das roturas irreparáveis do manguito rotador. Na ausência desses estudos, resta a comparação entre séries de casos tratadas por técnicas diferentes. Uma ressalva importante ao se realizar esse tipo de comparação é que as populações podem não ser semelhantes, levando a viés de seleção. Ao comparamos nossa série com estudos que avaliam a reconstrução da cápsula superior, observamos resultados inferiores aos reportados por Mihata et al. $^{22}$ e Burkhart et al., ${ }^{23}$ com respectivamente 92,9 e 89 pontos pela escala da ASES. Entretanto, Pennington et al. ${ }^{24}$ reportam 82 pontos, Denard et al. $.^{25} 77,5$ pontos e Hirahara et al. ${ }^{26} 70,7$ pontos pela mesma escala clínica, valores que se assemelham aos nossos. A artroplastia reversa, outra opção para o tratamento das roturas irreparáveis do manguito rotador, leva a uma média de 72,2 pontos pela escala da ASES e de 26,9 pela UCLA, de acordo com a revisão sistemática de Petrillo et al., ${ }^{27}$ resultados também próximos aos nossos. Entretanto, este estudo inclui tanto pacientes com roturas irreparáveis como aqueles com artropatia do manguito rotador, além de utilizar uma amostra de pacientes com idade mais avançada. Maillot et al., ${ }^{8}$ em uma metanálise envolvendo 20 estudos e 1.233 pacientes, compararam as diferentes formas de tratamento para as roturas grandes ou extensas do manguito rotador (tratamento conservador, desbridamento, reparo parcial, reparo completo, transferência do grande dorsal, uso de "patches", plasma rico em plaquetas e artroplastia reversa). Observaram que o único tratamento que demonstrou superioridade frente aos demais foi a transferência do grande dorsal. Tratamento conservador, reparo parcial, reparo completo e artroplastia reversa não diferiram nos resultados clínicos.

Observamos que $97 \%$ dos pacientes de nossa série referem estar satisfeitos com o procedimento, valor superior ao reportado por Cuff et al. ${ }^{14}$ (82\%) e Heuberer et al. ${ }^{28}(86 \%)$. Entretanto, em nosso estudo, o dado foi obtido por um subdomínio da escala da UCLA, e não por uma pergunta específica sobre satisfação, o que talvez justifique a diferença.

A flexão anterior ativa maior melhorou de maneira significativa em nossa série, e os pacientes com arco de movimento $\geq 150^{\circ}$ passaram de 36,4 para $75,8 \%$ da amostra. Esta melhora é compatível com os resultados de outros autores. ${ }^{6,16,21} \mathrm{Da}$ mesma maneira, a força de flexão anterior ativa apresentou melhora estatisticamente significante, com $66,7 \%$ dos pacientes apresentando força normal ou boa após o procedimento, contra $27,3 \%$ antes dele. Outros autores já demonstraram a melhora da força após o reparo parcial do manguito rotador. ${ }^{6,15,16,19,20}$ Cabe ressaltar que o reparo artroscópico do manguito rotador é efetivo em reverter quadro de pseudoparalisia do ombro, ${ }^{29}$ e que os bons resultados obtidos após o reparo de roturas extensas são mantidos no médio e longo prazo. ${ }^{30}$ Entretanto, é importante destacar que os resultados não são excelentes, e que $24,2 \%$ da amostra não consegue elevar $>150^{\circ}$, e $33,3 \%$ mantêm fraqueza importante ao final do seguimento clínico.

Nosso estudo possui algumas limitações. O desenho utilizado foi retrospectivo e não comparativo. Entretanto, os dados foram colhidos de maneira prospectiva, o que diminui o viés de aferição. Além disso, uma revisão sistemática recente 
demonstrou que a maioria dos estudos sobre o tema são retrospectivos. $^{7}$ A casuística de 33 pacientes também pode ser considerada pequena, mas estudos sobre o mesmo tema lidam com amostras de 14 a 73 reparos parciais. ${ }^{6,7}$ A força de flexão anterior, avaliada por nós, também não reflete toda a biomecânica do ombro, e a mensuração das forças de abdução, rotação lateral e medial poderia acrescentar informações importantes na análise funcional pré- e pós-operatória. As avaliações referentes a força, amplitude de movimento e satisfação foram realizadas por meio de subdomínios da escala da UCLA, e não de maneira mais detalhada e objetiva, o que também pode ser motivo de viés. Por fim, não avaliamos os pacientes com métodos de imagem após o reparo. Malahias et al. ${ }^{7}$ demonstraram que $49 \%$ dos pacientes submetidos ao reparo parcial apresentam rerrotura.

Os dados apresentados por nós mostram que o reparo parcial do manguito rotador é um procedimento eficaz no tratamento das roturas extensas do manguito rotador. A decisão entre as diferentes opções cirúrgicas deve levar em consideração a experiência do cirurgião e características individuais dos pacientes. Estudos randomizados são necessários para elucidar qual a melhor forma de tratamento para as roturas extensas e irreparáveis do manguito rotador.

\section{Conclusão}

O reparo parcial nas roturas irreparáveis do manguito rotador leva a melhora significativa de acordo com as escalas da ASES e UCLA, de modo estatisticamente significante e clinicamente relevante.

\section{Suporte Financeiro}

Não houve apoio financeiro de fontes públicas, comerciais, ou sem fins lucrativos.

\section{Conflito de Interesses}

Os autores declaram não haver conflito de interesses.

\section{Referências}

1 Yamamoto A, Takagishi K, Osawa T, et al. Prevalence and risk factors of a rotator cuff tear in the general population. J Shoulder Elbow Surg 2010;19(01):116-120

2 Malavolta EA, Gracitelli MEC, Assunção JH, Pinto GMR, da Silveira AZF, Ferreira AA. Shoulder disorders in an outpatient clinic: an epidemiological study. Acta Ortop Bras 2017;25(03):78-80

3 Malavolta EA, Assunção JH, Beraldo RA, Pinto GMR, Gracitelli MEC, Ferreira Neto AA. Rotator cuff repair in the Brazilian Unified Health System: Brazilian trends from 2003 to 2015. Rev Bras Ortop 2017;52(04):501-505

4 Kooistra B, Gurnani N, Weening A, van den Bekerom M, van Deurzen D. Low level of evidence for all treatment modalities for irreparable posterosuperior rotator cuff tears. Knee Surg Sports Traumatol Arthrosc 2019;27(12):4038-4048

5 Burkhart SS. Reconciling the paradox of rotator cuff repair versus debridement: a unified biomechanical rationale for the treatment of rotator cuff tears. Arthroscopy 1994;10(01):4-19

6 Burkhart SS, Nottage WM, Ogilvie-Harris DJ, Kohn HS, Pachelli A. Partial repair of irreparable rotator cuff tears. Arthroscopy 1994; 10(04):363-370
7 Malahias MA, Kostretzis L, Chronopoulos E, Brilakis E, Avramidis G, Antonogiannakis E. Arthroscopic partial repair for massive rotator cuff tears: does it work? A systematic review. Sports Med Open 2019;5(01):13

8 Maillot C, Martellotto A, Demezon H, Harly E, Le Huec J-C. Multiple Treatment Comparisons for Large and Massive Rotator Cuff Tears: A Network Meta-analysis. Clin J Sport Med 2019;•••;. Doi: 10.1097/JSM.0000000000000786

9 Knaut LA, Moser ADL, Melo SdeA, Richards RR. Translation and cultural adaptation to the portuguese language of the American Shoulder and Elbow Surgeons Standardized Shoulder assessment form (ASES) for evaluation of shoulder function. Rev Bras Reumatol 2010;50(02):176-189

10 Oku EC, Andrade AP, Stadiniky SP, Carrera EF. Tradução e adaptação cultural do Modified-University of California at Los Angeles Shoulder Rating Scale para a língua portuguesa. Rev Bras Reumatol 2006;46(04):246-252

11 Fuchs B, Weishaupt D, Zanetti M, Hodler J, Gerber C. Fatty degeneration of the muscles of the rotator cuff: assessment by computed tomography versus magnetic resonance imaging. J Shoulder Elbow Surg 1999;8(06):599-605

12 Boileau P, Brassart N, Watkinson DJ, Carles M, Hatzidakis AM, Krishnan SG. Arthroscopic repair of full-thickness tears of the supraspinatus: does the tendon really heal? J Bone Joint Surg Am 2005;87(06):1229-1240

13 Cvetanovich GL, Gowd AK, Liu JN, et al. Establishing clinically significant outcome after arthroscopic rotator cuff repair. J Shoulder Elbow Surg 2019;28(05):939-948

14 Cuff DJ, Pupello DR, Santoni BG. Partial rotator cuff repair and biceps tenotomy for the treatment of patients with massive cuff tears and retained overhead elevation: midterm outcomes with a minimum 5 years of follow-up. J Shoulder Elbow Surg 2016;25(11):1803-1809

15 Holtby R, Razmjou H. Relationship between clinical and surgical findings and reparability of large and massive rotator cuff tears: a longitudinal study. BMC Musculoskelet Disord 2014;15:180

16 Franceschi F, Papalia R, Vasta S, Leonardi F, Maffulli N, Denaro V. Surgical management of irreparable rotator cuff tears. Knee Surg Sports Traumatol Arthrosc 2015;23(02):494-501

17 Iagulli ND, Field LD, Hobgood ER, Ramsey JR, Savoie FH III. Comparison of partial versus complete arthroscopic repair of massive rotator cuff tears. Am J Sports Med 2012;40(05):1022-1026

18 Berth A, Neumann W, Awiszus F, Pap G. Massive rotator cuff tears: functional outcome after debridement or arthroscopic partial repair. J Orthop Traumatol 2010;11(01):13-20

19 Godenèche A, Freychet B, Lanzetti RM, Clechet J, Carrillon Y, Saffarini M. Should massive rotator cuff tears be reconstructed even when only partially repairable? Knee Surg Sports Traumatol Arthrosc 2017;25(07):2164-2173

20 Kim SJ, Lee IS, Kim SH, Lee WY, Chun YM. Arthroscopic partial repair of irreparable large to massive rotator cuff tears. Arthroscopy 2012;28(06):761-768

21 Mori D, Funakoshi N, Yamashita F. Arthroscopic surgery of irreparable large or massive rotator cuff tears with low-grade fatty degeneration of the infraspinatus: patch autograft procedure versus partial repair procedure. Arthroscopy 2013;29(12):1911-1921

22 Mihata T, Lee TQ, Watanabe C, et al. Clinical results of arthroscopic superior capsule reconstruction for irreparable rotator cuff tears. Arthroscopy 2013;29(03):459-470

23 Burkhart SS, Hartzler RU. Superior Capsular Reconstruction Reverses Profound Pseudoparalysis in Patients With Irreparable Rotator Cuff Tears and Minimal or No Glenohumeral Arthritis. Arthroscopy 2019;35(01):22-28

24 Pennington WT, Bartz BA, Pauli JM, Walker CE, Schmidt W. Arthroscopic Superior Capsular Reconstruction With Acellular Dermal Allograft for the Treatment of Massive Irreparable Rotator Cuff Tears: Short-Term Clinical Outcomes and the Radiographic Parameter of Superior Capsular Distance. Arthroscopy 2018;34(06):1764-1773 
25 Denard PJ, Brady PC, Adams CR, Tokish JM, Burkhart SS. Preliminary Results of Arthroscopic Superior Capsule Reconstruction with Dermal Allograft. Arthroscopy 2018;34(01):93-99

26 Hirahara AM, Andersen WJ, Panero AJ. Superior Capsular Reconstruction: Clinical Outcomes After Minimum 2-Year Follow-Up. Am J Orthop 2017;46(06):266-278

27 Petrillo S, Longo UG, Papalia R, Denaro V. Reverse shoulder arthroplasty for massive irreparable rotator cuff tears and cuff tear arthropathy: a systematic review. Musculoskelet Surg 2017; 101(02):105-112

28 Heuberer PR, Kölblinger R, Buchleitner S, et al. Arthroscopic management of massive rotator cuff tears: an evaluation of debridement, complete, and partial repair with and without force couple restoration. Knee Surg Sports Traumatol Arthrosc 2016;24 (12):3828-3837

29 Miyazaki AN, Fregoneze M, Santos PD, et al. Avaliação funcional do reparo artroscópico da lesão do manguito rotador em pacientes com pseudoparalisia. Rev Bras Ortop 2014;49(02): 178-182

30 Miyazaki AN, Santos PD, da Silva LA, do Val Sella G, Checchia SL, Yonamine AM. Are the good functional results from arthroscopic repair of massive rotator cuff injuries maintained over the long term? Rev Bras Ortop 2015;51(01):40-44 